\title{
Tangent Bundle of Pseudo-sphere and Ruled Surfaces in Minkowski 3-space
}

\author{
Murat BEKAR ${ }^{1}$, Fouzi HATHOUT ${ }^{\circledR 2}$ and Yusuf YAYLI ${ }^{3}$ \\ ${ }^{1}$ Gazi University, Department of Mathematics, 06900 Polatli/Ankara-Turkey. \\ 2 Department of Mathematics, Saïda University, 20000 Saïda, Algeria. \\ ${ }^{3}$ Department of Mathematics, Ankara University, 06100 Ankara, Turkey. \\ ${ }^{1}$ muratbekar@gazi.edu.tr, ${ }^{2}$ f.hathout@gmail.com, ${ }^{3}$ yayli@science.ankara.edu.tr
}

\begin{abstract}
According to E. Study map in Minkowski space, we give in this present paper, a one-to-one correspondence between "the curves on tangent bundles of Lorentzian (or de Sitter) unit sphere $T \mathbb{S}_{1}^{2}$ and hyperbolic unit sphere $T \mathbb{H}^{2}$ " and "the space-like or time-like ruled surface in $\mathbb{E}_{1}^{3}$ ". In fact, we can consider each curve on $T \mathbb{S}_{1}^{2}$ or $T \mathbb{H}^{2}$ as a ruled surface in $\mathbb{E}_{1}^{3}$. Moreover, we study the relationships between the developability conditions of these corresponding ruled surfaces in $\mathbb{E}_{1}^{3}$ and their base and striction curves, and we show that if the curves on $T \mathbb{S}_{1}^{2}$ or $T \mathbb{H}^{2}$ are involute-evolute couples, then the corresponding ruled surfaces are developable.
\end{abstract}

Keywords: $\quad$ Tangent bundle, Unit dual pseudo-sphere, Minkowski space, E. Study map, Ruled surface, Legendre curve.

2010 MSC No: 53A35, 53A25.

\section{Introduction}

The author E. Study in [9], use the dual numbers introduced by W. K. Clifford, and the dual vectors in the geometry of lines and kinematics, he defined a map (which is called by his name) that corresponds to each unit dual vector a directed line in space.

In Minkowski context, the E. Study mapping can be stated as follows ([1]): The dual time-like and space-like unit vectors of the dual Lorentzian unit spheres $\mathbb{S}_{1}^{2}$ and the dualhyperbolic unit sphere $\mathcal{H}^{2}$ of the dual Lorentzian space $\mathbb{D}_{1}^{3}$ are in one-to-one correspondence with the directed time-like and space-like lines of the Lorentzian lines in $\mathbb{E}_{1}^{3}$, respectively. Hence, to any time-like or space-like curve on $\mathcal{S}_{1}^{2}$ corresponds space-like or time-like ruled surface of $\mathbb{E}_{1}^{3}$, and to a smooth curve on $\mathcal{H}^{2}$ corresponds a time-like ruled surface in $\mathbb{E}_{1}^{3}$, respectively.

We extend the E. Study correspondence map to the tangent bundles of the Lorentzian (or de Sitter) unit sphere $\mathbb{S}_{1}^{2}$ and hyperbolic unit sphere $\mathbb{H}^{2}$ as: There exists a one-to-one correspondence between any smooth curve $\Gamma$ on $T \mathbb{S}_{1}^{2}$ and a space-like or time-like ruled surface in $\mathbb{E}_{1}^{3}$, and a one-to-one correspondence between any smooth curve $\Gamma$ on $T \mathbb{H}^{2}$ and a time-like ruled surface in $\mathbb{E}_{1}^{3}$, respectively, see Propositions 3 and 3 . We devote our study to the developability conditions of these ruled surfaces (with different causal characters) depending to their corresponding smooth curves on $T \mathbb{S}_{1}^{2}$ and $T \mathbb{H}^{2}$ to be Legendre or involute-evolute curve couples (see [4], 11], 12]).

The paper is presented as follows: In section 2, we give some notions and definitions of "the tangent bundles of Lorentzian (or de Sitter) unit sphere $\mathbb{S}_{1}^{2}$ and hyperbolic unit sphere $\mathbb{H}^{2}$ ", "ruled surfaces" and "Legendre curves". In

\footnotetext{
${ }^{*}$ Corresponding author. Fouzi HATHOUT ${ }^{1}$ f.hathout@gmail.com
} 
Section 3, we recall the definitions and some properties of dual numbers, and give a correspondence between "the curves on $T \mathbb{S}_{1}^{2}$ and $T \mathbb{H}^{2}$ " and "the ruled surfaces in $\mathbb{E}_{1}^{3}$ ". The developability conditions of these ruled surfaces are also considered. Finally, in Section 4, some properties of the developability and the relationship between the base and striction curves of the corresponding ruled surfaces in $\mathbb{E}_{1}^{3}$ to the curves in $T \mathbb{S}_{1}^{2}$ and $T \mathbb{H}^{2}$ are studied, and some examples are given.

\section{Preliminaries}

Let $\mathbb{E}_{1}^{3}$ be a 3-dimensional Minkowski space endowed with the Lorentz scalar product given by

$$
\langle,\rangle_{1}=d x_{1}^{2}+d x_{2}^{2}-d x_{3}^{2}
$$

where $x=\left(x_{1}, x_{2}, x_{3}\right)$ is a standard rectangular coordinate system of $\mathbb{E}_{1}^{3}$. An arbitrary vector $x$ in $\mathbb{E}_{1}^{3}$ can be called by one of the following three Lorentzian causal characters:

(i) Space-like if $\langle x, x\rangle_{1}>0$ or $x=0$,

(ii) Time-like if $\langle x, x\rangle_{1}<0$,

(iii) Light-like (or null) if $\langle x, x\rangle_{1}=0$ for $x \neq 0$.

The norm of $x$ is denoted by $\|x\|_{1}=\sqrt{\left|\langle x, x\rangle_{1}\right|}$, and two vectors $x$ and $y$ in $\mathbb{E}_{1}^{3}$ are said to be orthogonal if $\langle x, y\rangle_{1}=0$. The Lorentz vector product of any two vectors $x=\left(x_{1}, x_{2}, x_{3}\right)$ and $y=\left(y_{1}, y_{2}, y_{3}\right)$ in $\mathbb{E}_{1}^{3}$ is

$$
x \wedge_{1} y=\left(x_{3} y_{2}-x_{2} y_{3}, x_{1} y_{3}-x_{3} y_{1}, x_{1} y_{2}-x_{2} y_{1}\right),
$$

see 7 .

Lorentzian (or de Sitter) unit sphere and hyperbolic unit sphere in $\mathbb{E}_{1}^{3}$, see Fig. 1, are given, respectively, by

$$
\begin{aligned}
\mathbb{S}_{1}^{2} & =\left\{x=\left(x_{1}, x_{2}, x_{3}\right) \in \mathbb{E}_{1}^{3}:\langle x, x\rangle_{1}=1\right\}, \\
\mathbb{H}^{2} & =\left\{x=\left(x_{1}, x_{2}, x_{3}\right) \in \mathbb{E}_{1}^{3}:\langle x, x\rangle_{1}=-1\right\},
\end{aligned}
$$

see [7, 12. Tangent bundles of $\mathbb{S}_{1}^{2}$ and $\mathbb{H}^{2}$ are, respectively,

$$
\begin{aligned}
T \mathbb{S}_{1}^{2} & =\left\{(\gamma, v) \in \mathbb{S}_{1}^{2} \times \mathbb{E}_{1}^{3}:\langle\gamma, v\rangle_{1}=0\right\} \\
T \mathbb{H}^{2} & =\left\{(\gamma, v) \in \mathbb{H}^{2} \times \mathbb{E}_{1}^{3}:\langle\gamma, v\rangle_{1}=0\right\} .
\end{aligned}
$$

And the unit tangent bundles of $\mathbb{S}_{1}^{2}$ and $\mathbb{H}^{2}$ are, respectively,

$$
\begin{aligned}
U T \mathbb{S}_{1}^{2} & =\left\{(\gamma, v) \in \mathbb{S}_{1}^{2} \times \mathbb{E}_{1}^{3}:\langle\gamma, v\rangle_{1}=0 \text { and }\langle v, v\rangle_{1}= \pm 1\right\}, \\
U T \mathbb{H}^{2} & =\left\{(\gamma, v) \in \mathbb{H}^{2} \times \mathbb{E}_{1}^{3}:\langle\gamma, v\rangle_{1}=0 \text { and }\langle v, v\rangle_{1}=1\right\} .
\end{aligned}
$$

A curve $\gamma: I \subset \mathbb{R} \rightarrow \mathbb{E}_{1}^{3}$ with arc-length parameter $s$ can be locally space-like, time-like or light-like (or null), if all of its velocity vectors $\gamma^{\prime}(s)$ are respectively space-like, time-like or light-like (or null). A space-like (resp. time-like) curve $\gamma(s)$ is called a unit speed curve if $\left\langle\gamma^{\prime}, \gamma_{1}^{\prime}\right\rangle_{1}=1\left(\operatorname{resp} .\left\langle\gamma^{\prime}, \gamma_{1}^{\prime}\right\rangle_{1}=-1\right)$, see [5].

Let $\gamma: I \subset \mathbb{R} \rightarrow \mathbb{E}_{1}^{3}$ be a non-null speed curve parameterized by arc-length parameter $s$ with Frenet frame apparatus $\{T, N, B, \kappa, \tau\}$. If $\gamma$ is a space-like (resp. time-like) curve in $\mathbb{E}_{1}^{3}$, the moving Frenet formulae are

$$
\begin{gathered}
\left(\begin{array}{l}
T^{\prime} \\
N^{\prime} \\
B^{\prime}
\end{array}\right)=\left(\begin{array}{ccc}
0 & \kappa & 0 \\
-\varepsilon_{1} \kappa & 0 & \tau \\
0 & \varepsilon_{1} \tau & 0
\end{array}\right)\left(\begin{array}{c}
T \\
N \\
B
\end{array}\right) \\
\text { resp. } \left.\left(\begin{array}{c}
T^{\prime} \\
N^{\prime} \\
B^{\prime}
\end{array}\right)=\left(\begin{array}{ccc}
0 & \kappa & 0 \\
\kappa & 0 & \tau \\
0 & -\tau & 0
\end{array}\right)\left(\begin{array}{c}
T \\
N \\
B
\end{array}\right)\right)
\end{gathered}
$$

where $\kappa$ and $\tau$ are, respectively, the curvature and torsion of the curve $\gamma$, and $\langle T, T\rangle_{1}=1\left(\operatorname{resp} .\langle T, T\rangle_{1}=-1\right)$, $\langle N, N\rangle_{1}=\varepsilon_{1}= \pm 1$ (resp. $\left.\langle N, N\rangle_{1}=1\right),\langle B, B\rangle_{1}=-\varepsilon_{1}\left(\operatorname{resp} .\langle B, B\rangle_{1}=1\right)$, see [5]. The curve $\Gamma=(\gamma, v)$ is said to be a Legendre curve in $T \mathbb{S}_{1}^{2}\left(\right.$ resp. $\left.T \mathbb{H}^{2}\right)$ if the pair $(\gamma, v)$ satisfy

$$
\left\langle\gamma^{\prime}(s), v(s)\right\rangle_{1}=0 \text { for all } s \in I \text {. }
$$




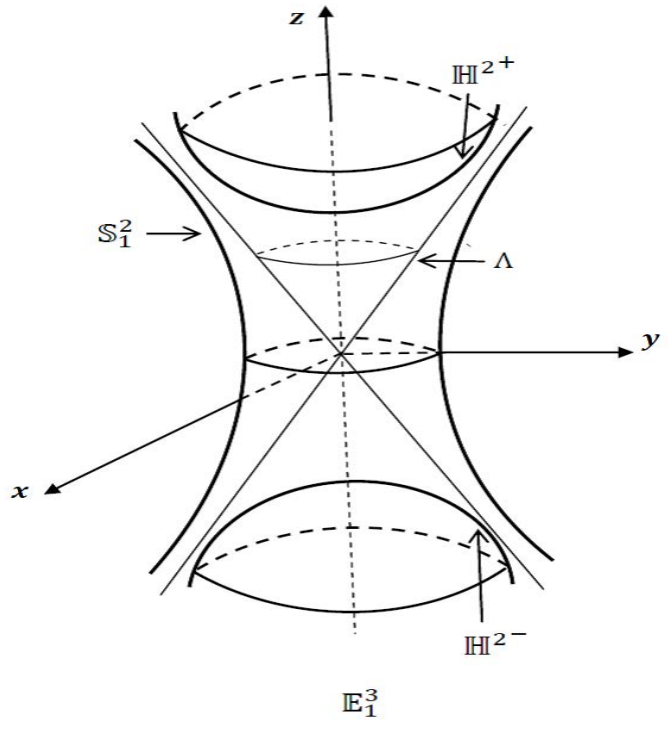

Fig. 1: Lorentzian unit sphere $\mathbb{S}_{1}^{2}$, Hyperbolic unit sphere $\mathbb{H}^{2}=\mathbb{H}^{2+} \cup \mathbb{H}^{2-}$ and light-like (or null) cone $\Lambda$ in $\mathbb{E}_{1}^{3}$, where $\mathbb{H}^{2^{+}}$and $\mathbb{H}^{2^{-}}$denote, respectively, the future-pointing and past-pointing hyperbolic unit spheres.

On the other hand, let $I$ and $J$ be open intervals in $\mathbb{R}, \beta$ be a curve of $I$ into $\mathbb{E}_{1}^{3}$ and $\gamma$ be a vector field along $\beta$. Then, the ruled surface $\Phi(s, u)$ is defined in $\mathbb{E}_{1}^{3}$ by the parametrization given as

$$
\Phi(s, u)=\beta(s)+u \gamma(s) ; \quad s \in I \subset \mathbb{R}, \quad u \in J \subset \mathbb{R} .
$$

Here, the curves $\beta$ and $\gamma$ are called, respectively, the base curve and the director curve. The striction curve of the ruled surface $\Phi(s, u)$ is defined by

$$
\bar{\beta}(s)=\beta(s)-\frac{\left\langle T_{\beta}, \gamma^{\prime}(s)\right\rangle_{1}}{\left\langle\gamma^{\prime}(s), \gamma^{\prime}(s)\right\rangle_{1}} \gamma^{\prime}(s),
$$

where $T_{\beta}=\beta^{\prime}(s)$. If $\left\langle T_{\beta}, \gamma^{\prime}(s)\right\rangle_{1}=0$, then the striction curve $\bar{\beta}$ coincides with the base curve $\beta$ of $\Phi(s, u)$, see [6].

\section{Unit dual pseudo-sphere and ruled surfaces in $\mathbb{E}_{1}^{3}$}

Let $a$ and $a^{*}$ be any two real numbers and $\varepsilon \neq 0$ satisfying $\varepsilon^{2}=0$. Then, $A=a+\varepsilon a^{*}$ is called a dual number. Here, $a$ and $a^{*}$ are, respectively, the non-dual and dual parts of $A$ and $\varepsilon$ is the dual unit satisfying $r \varepsilon=\varepsilon r$ for all $r \in \mathbb{R}$. The set

$$
\mathbb{D}=\left\{A=a+\varepsilon a^{*}:\left(a, a^{*}\right) \in \mathbb{R} \times \mathbb{R}, \quad \varepsilon \neq 0 \text { and } \varepsilon^{2}=0\right\}
$$

of dual numbers is a commutative ring over the real number field according to the addition and multiplication operations

$$
\left(a+\varepsilon a^{*}\right)+\left(b+\varepsilon b^{*}\right)=(a+b)+\varepsilon\left(a^{*}+b^{*}\right)
$$

and

$$
\left(a+\varepsilon a^{*}\right)\left(b+\varepsilon b^{*}\right)=a b+\varepsilon\left(a b^{*}+a^{*} b\right),
$$

respectively. The zero divisors of this field are the numbers $\varepsilon a^{*}$ for all $a^{*} \in \mathbb{R}$, see [1, 10].

The set

$$
\mathbb{D}^{3}=\left\{\vec{A}=\left(A_{1}, A_{2}, A_{3}\right): A_{i}=a_{i}+\varepsilon a_{i}^{*} \in \mathbb{D}, \quad 1 \leq i \leq 3\right\}
$$

is a module over the ring $\mathbb{D}$ and is called the dual-space (or $\mathbb{D}$-module). The elements of $\mathbb{D}^{3}$ are the dual vectors. A dual vector $\vec{A}=\left(A_{1}, A_{2}, A_{3}\right)$ can be written in dual form as

$$
\vec{A}=\vec{a}+\varepsilon \overrightarrow{a^{*}}
$$


where $\vec{a}=\left(a_{1}, a_{2}, a_{3}\right)$ and $\overrightarrow{a^{*}}=\left(a_{1}^{*}, a_{2}^{*}, a_{3}^{*}\right)$ are real vectors in $\mathbb{E}^{3}$. Here, $\vec{a}$ is the non-dual part and $\overrightarrow{a^{*}}$ is the $d u a l$ part of $\vec{A}$. Addition and Lorentzian inner-product of any dual vectors $\vec{A}=\vec{a}+\varepsilon \vec{a}^{*}$ and $\vec{B}=\vec{b}+\varepsilon \overrightarrow{b^{*}}$ are, respectively, defined by

$$
\vec{A}+{ }_{1} \vec{B}=(\vec{a}+\vec{b})+\varepsilon\left(\overrightarrow{a^{*}}+\overrightarrow{b^{*}}\right)
$$

and

$$
\langle\vec{A}, \vec{B}\rangle_{1}=\langle\vec{a}, \vec{b}\rangle_{1}+\varepsilon\left(\left\langle\overrightarrow{a^{*}}, \vec{b}\right\rangle_{1}+\left\langle\vec{a}, \overrightarrow{b^{*}}\right\rangle_{1}\right) .
$$

If $\langle\vec{A}, \vec{B}\rangle_{1}=0$, the dual vectors $\vec{A}$ and $\vec{B}$ are said to be perpendicular in sense of Lorentz, see [11].

A dual Lorentzian vector $\vec{A}=\vec{a}+\varepsilon \overrightarrow{a^{*}}$ is said to be time-like if $\vec{a}$ is time-like (i.e., $\langle\vec{a}, \vec{a}\rangle_{1}<0$ or $\vec{a}=0$ ), spacelike if $\vec{a}$ is space-like (i.e., $\langle\vec{a}, \vec{a}\rangle_{1}>0$ ) and light-like (or null) if $\vec{a}$ is light-like (i.e., $\langle\vec{a}, \vec{a}\rangle_{1}=0$ and $\vec{a} \neq 0$ ). The set of all dual Lorentzian vectors is denoted by $\mathbb{D}_{1}^{3}$. We call the set of all dual Lorentzian light-like (or null) vectors as the light-like (or null) cone in $\mathbb{D}_{1}^{3}$. Moreover, a dual time-like vector $\vec{A}=\vec{a}+\varepsilon \overrightarrow{a^{*}}=\left(a_{1}, a_{2}, a_{3}\right)+\varepsilon\left(a_{1}^{*}, a_{2}^{*}, a_{3}^{*}\right)$ is said to be future-pointing (resp. past-pointing) if and only if $a_{3}>0$ (resp. $a_{3}<0$ ), see [11].

Similarly, an arbitrary dual Lorentzian space curve

$$
\widehat{A}: I \subset \mathbb{R} \rightarrow \mathbb{D}_{1}^{3}
$$

defined by

$$
t \mapsto \widehat{A}(t)=\vec{a}(t)+\varepsilon \overrightarrow{a^{*}}(t)
$$

is said to be time-like if its velocity vector $\alpha^{\prime}(t)=d \alpha / d t$ is time-like, space-like if its velocity vector is space-like and light-like (or null) if its velocity vector is light-like (or null) for all $t \in \mathbb{R}$. Also, a dual time-like space curve $\widehat{A}(t)=\vec{a}(t)+\varepsilon \vec{a}^{*}(t)$ is said to be future-pointing (resp. past-pointing) if $\vec{a}(t)$ is future-pointing (resp. past-pointing). In this study we consider only the non-null dual vectors and curves, see [8].

If each real valued functions $a_{i}(t)$ and $a_{i}^{*}(t)$ for $1 \leq i \leq 3$ are differentiable, the dual space curve $\widehat{A}(t)=$ $\vec{a}(t)+\varepsilon \overrightarrow{a^{*}}(t)$ is differentiable in $\mathbb{D}_{1}^{3}$, where $\vec{a}=\left(a_{1}, a_{2}, a_{3}\right) \in \mathbb{E}_{1}^{3}$ and $\overrightarrow{a^{*}}=\left(a_{1}^{*}, a_{2}^{*}, a_{3}^{*}\right) \in \mathbb{E}_{1}^{3}$.

The Lorentzian cross-product of the dual Lorentzian vectors $\vec{A}=\vec{a}+\varepsilon \overrightarrow{a^{*}}$ and $\vec{B}=\vec{b}+\varepsilon b^{*}$ is defined by

$$
\vec{A} \wedge_{1} \vec{B}=\vec{a} \wedge_{1} \vec{b}+\varepsilon\left(\vec{a} \wedge_{1} \overrightarrow{b^{*}}+\overrightarrow{a^{*}} \wedge_{1} \vec{b}\right)
$$

The Lorentzian (or pseudo) norm of a non-null dual Lorentzian vector $\vec{A}=\vec{a}+\varepsilon \vec{a}^{*}$ is defined to be

$$
\|\vec{A}\|_{1}=\sqrt{\left|\langle\vec{a}, \vec{a}\rangle_{1}\right|}+\varepsilon \frac{\left\langle\vec{a}, \overrightarrow{a^{*}}\right\rangle_{1}}{\sqrt{\left|\langle\vec{a}, \vec{a}\rangle_{1}\right|}}
$$

where $|$.$| denotes the absolute value of a real number. If \|\vec{A}\|_{1}=1$ (that is, $\langle\vec{a}, \vec{a}\rangle_{1}= \pm 1$ and $\left\langle\vec{a}, \overrightarrow{a^{*}}\right\rangle_{1}=0$ ), then $\vec{A}$ is said to be a unit. We denote the set of all unit dual Lorentzian vectors by $\widehat{\mathbb{D}}_{1}^{3}$. The set

$$
\mathcal{H}^{2}=\left\{\vec{A}=\vec{a}+\varepsilon \overrightarrow{a^{*}} \in \widehat{\mathbb{D}}_{1}^{3}:\langle\vec{a}, \vec{a}\rangle_{1}=-1+\varepsilon 0 \quad \text { and } \quad\left\langle\vec{a}, \overrightarrow{a^{*}}\right\rangle_{1}=0\right\}
$$

of all unit dual time-like vectors is called the dual hyperbolic unit sphere, and the set

$$
\mathcal{S}_{1}^{2}=\left\{\vec{A}=\vec{a}+\varepsilon \vec{a}^{*} \in \widehat{\mathbb{D}}_{1}^{3}:\langle\vec{a}, \vec{a}\rangle_{1}=1+\varepsilon 0 \quad \text { and }\left\langle\vec{a}, \overrightarrow{a^{*}}\right\rangle_{1}=0\right\}
$$

of all unit dual space-like vectors is called the dual Lorentzian unit sphere, see [12].

There are two components of the dual hyperbolic unit sphere $\mathcal{H}^{2}$. One of them is the set

$$
\mathcal{H}^{2^{+}}=\left\{\vec{A}=\vec{a}+\varepsilon \overrightarrow{a^{*}} \in \mathcal{H}^{2}: \vec{a} \in \mathbb{E}_{1}^{3} \text { is a future-pointing time-like unit vector }\right\},
$$

known as the future-pointing dual hyperbolic unit sphere. The other one is the set

$$
\mathcal{H}^{2^{-}}=\left\{\vec{A}=\vec{a}+\varepsilon \vec{a}^{*} \in \mathcal{H}_{0}^{2}: \vec{a} \in \mathbb{E}_{1}^{3} \text { is a past-pointing time-like unit vector }\right\},
$$


known as the past-pointing dual hyperbolic unit sphere. Hence,

$$
\mathcal{H}^{2}=\mathcal{H}^{2^{+}} \cup \mathcal{H}^{2-} \text {. }
$$

As a result, the following proposition can be given by using the Eq. (1), Eq. (7) and Eq. (8):

The maps

$$
\begin{aligned}
& T \mathbb{S}_{1}^{2} \rightarrow \mathcal{S}_{1}^{2} \\
& \Gamma=(\gamma, \nu) \mapsto \vec{\Gamma}=\gamma+\varepsilon \nu \\
& T \mathbb{H}^{2} \rightarrow \mathcal{H}^{2} \\
& \Gamma=(\gamma, \nu) \mapsto \vec{\Gamma}=\gamma+\varepsilon \nu
\end{aligned}
$$

are isomorphisms, i.e. $T \mathbb{S}_{1}^{2} \cong \mathcal{S}_{1}^{2}$ and $T \mathbb{H}^{2} \cong \mathcal{H}^{2}$

A directed time-like (resp. space-like) line in $\mathbb{E}_{1}^{3}$ is uniquely defined by any different two points $p$ and $q$ on it. If $t$ is any non-zero constant, the parametric equation of the line is

$$
\vec{q}=\vec{p}+t \vec{x}
$$

The vector defined by

$$
\overrightarrow{x^{*}}=\vec{p} \wedge_{1} \vec{x}=\vec{q} \wedge_{1} \vec{x}
$$

is the moment of $\vec{x}$ with respect to the origin $O$. This means that the direction vector $\vec{x}$ of the directed time-like (resp. space-like) line and its moment vector $\overrightarrow{x^{*}}$ are independent of the choice of the points $p$ and $q$ on the line. However, the vectors $\vec{x}$ and $\overrightarrow{x^{*}}$ are not independent of one another because they satisfy the equations

$$
\langle\vec{x}, \vec{x}\rangle_{1}=-1\left(\operatorname{resp} .\langle\vec{x}, \vec{x}\rangle_{1}=1\right) \text { and }\left\langle\vec{x}, \overrightarrow{x^{*}}\right\rangle_{1}=0 .
$$

Theorem 3.1 ([1] , E. Study Map). There exists a one-to-one correspondence between the directed time-like (resp. space-like) lines of $\mathbb{E}_{1}^{3}$ and an ordered pairs of vectors $\left(\vec{a}, \overrightarrow{a^{*}}\right)$ such that $\langle\vec{a}, \vec{a}\rangle_{1}=-1\left(\right.$ resp. $\left.\langle\vec{a}, \vec{a}\rangle_{1}=1\right)$ and $\left\langle\vec{a}, \overrightarrow{a^{*}}\right\rangle_{1}=0$.

Corollary 3.2. Let $\vec{A}=\vec{a}+\varepsilon \vec{a}^{*}$ be a unit dual Lorentzian vector (i.e., $\vec{A} \in \widehat{\mathbb{D}}_{1}^{3}$ so $\langle\vec{a}, \vec{a}\rangle_{1}= \pm 1$ and $\left\langle\vec{a}, \overrightarrow{a^{*}}\right\rangle_{1}=0$ ). Then, the parametric equation of the line $l_{\vec{A}}$ corresponding to $\vec{A}$ in $\mathbb{E}_{1}^{3}$ may be given as

$$
\vec{y}=\vec{z}+t \vec{a}, \quad t \text { is any non-zero constant }
$$

where $\vec{z}=\vec{a} \wedge_{1} \overrightarrow{a^{*}}, \overrightarrow{a^{*}}=\vec{z} \wedge_{1} \vec{a}\left(=\vec{y} \wedge_{1} \vec{a}\right)$ and the points $z$ and $y$ are any different two points on $l_{\vec{A}}$, see Fig. 2.

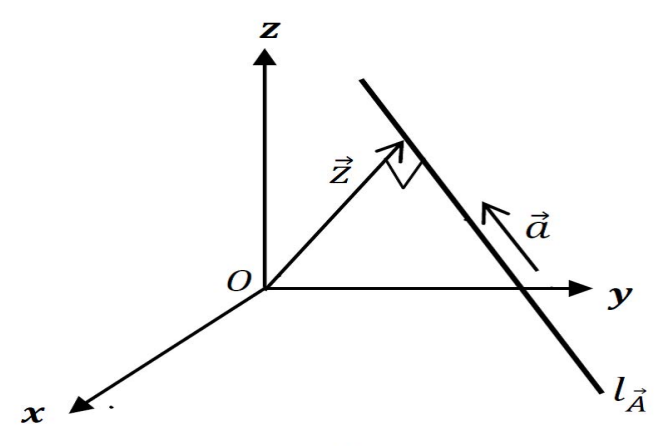

$\mathbb{E}_{1}^{3}$

Fig. 2: Unit dual Lorentzian vector $\vec{A}=\vec{a}+\varepsilon \overrightarrow{a^{*}}$ and the corresponding line $l_{\vec{A}}$ in $\mathbb{E}_{1}^{3}$.

A ruled surface is a surface generated by the motion of a straight line in $\mathbb{E}^{3}$. This straight line is the generator of the surface. We call a ruled surface as time-like if the induced metric on the surface is a Lorentzian metric (that means that the normal vector of the ruled surface at every point is a space-like vector) and as space-like if the induced metric on the surface is a positive defined Riemannian metric (that means that the normal vector of the ruled surface at every point is a time-like vector). 
Corollary 3.3. Let $l_{\vec{A}}$ be a line in $\mathbb{E}_{1}^{3}$ with the direction vector $\vec{a}$, and $\vec{p}$ be the position vector of any point on $l_{\vec{A}}$. Then, the unit dual Lorentzian vector corresponding to the line $l_{\vec{A}}$ can be given as

$$
\vec{A}=\vec{a}+\varepsilon\left(\vec{p} \wedge_{1} \vec{a}\right)=\vec{a}+\varepsilon \vec{a}^{*}
$$

Using the dual Lorentzian vector function

$$
\widehat{A}(t)=\vec{a}(t)+\varepsilon\left(\vec{p}(t) \wedge_{1} \vec{a}(t)\right)=\vec{a}(t)+\varepsilon \overrightarrow{a^{*}}(t)
$$

a ruled surface may be represented in $\mathbb{E}_{1}^{3}$ by

$$
\phi_{\widehat{A}}(t, u)=\vec{p}(t)+u \vec{a}(t)=\vec{a}(t) \wedge_{1} \overrightarrow{a^{*}}(t)+u \vec{a}(t) .
$$

A unit dual time-like (resp. space-like) vector $\vec{A}=\vec{a}+\varepsilon\left(\vec{p} \wedge_{1} \vec{a}\right)=\vec{a}+\varepsilon \vec{a}^{*}$ is a differentiable curve on $\mathcal{H}^{2}$ (resp. $\left.\mathcal{S}_{1}^{2}\right)$ and

$$
\begin{aligned}
\langle\vec{x}, \vec{x}\rangle_{1} & =\left\langle\vec{a}+\varepsilon\left(\vec{p} \wedge_{1} \vec{a}\right), \vec{a}+\varepsilon\left(\vec{p} \wedge_{1} \vec{a}\right)\right\rangle_{1} \\
& =\langle\vec{a}, \vec{a}\rangle_{1}+2 \varepsilon\left\langle\vec{a},\left(\vec{p} \wedge_{1} \vec{a}\right)\right\rangle_{1}+\varepsilon^{2}\left\langle\left(\vec{p} \wedge_{1} \vec{a}\right),\left(\vec{p} \wedge_{1} \vec{a}\right)\right\rangle_{1} \\
& =\langle\vec{a}, \vec{a}\rangle_{1}=-1(\operatorname{resp} .+1)
\end{aligned}
$$

thus having unit magnitude.

A ruled surface in $\mathbb{E}_{1}^{3}$ is said to be time-like if the normal of the surface is space-like at every point, and space-like if the normal of surface is time-like at every point.

A differentiable curve on $\mathcal{H}^{2}$ corresponds to a time-like ruled surface in $\mathbb{E}_{1}^{3}$ (see Fig. 3), and a time-like (resp. space-like) curve on $\mathcal{S}_{1}^{2}$ corresponds to a space-like (resp. time-like) ruled surface in $\mathbb{E}_{1}^{3}$ (see Fig. 4).

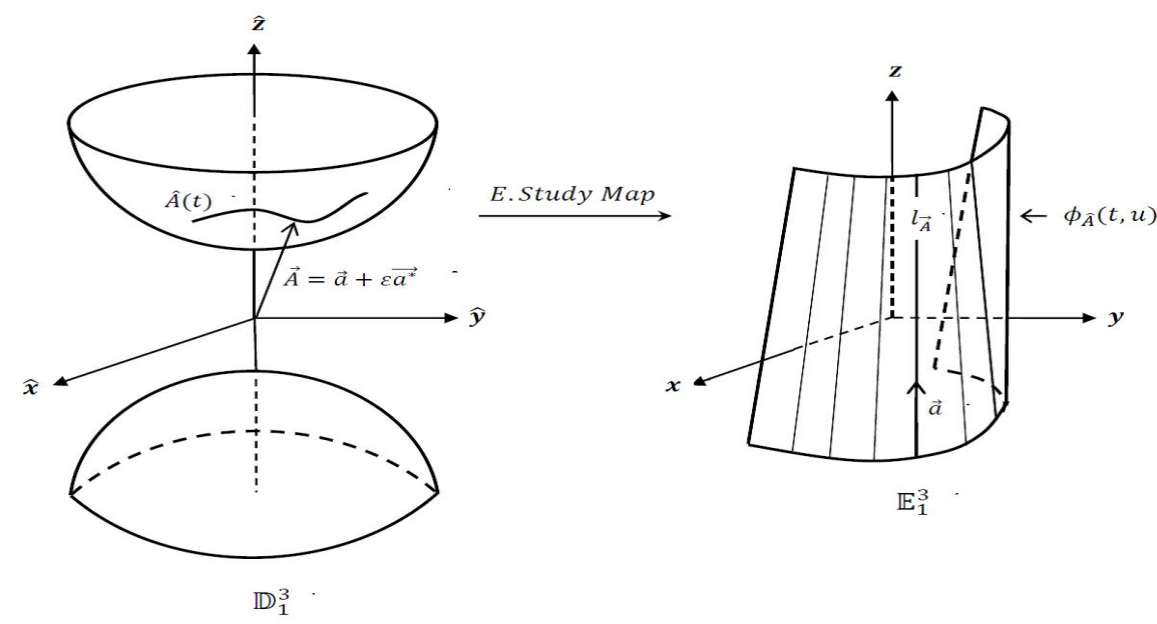

Fig. 3: The curve $\widehat{A}(t)$ on $\mathcal{H}^{2^{+}} \subset \mathcal{H}^{2}$ and the corresponding time-like ruled surface $\phi_{\widehat{A}}(t, u)$ in $\mathbb{E}_{1}^{3}$.

The map

$$
T \mathbb{H}^{2} \rightarrow \mathcal{H}^{2} \rightarrow \mathbb{E}_{1}^{3}
$$

defined by

$$
\Gamma(t)=(\gamma(t), \nu(t)) \mapsto \widehat{\Gamma}(t)=\vec{\gamma}(t)+\varepsilon \vec{\nu}(t) \rightarrow \phi_{\widehat{\Gamma}}(t, u)=\vec{\gamma}(t) \wedge_{1} \vec{\nu}(t)+u \vec{\gamma}(t)
$$

is an isomorphism, i.e. $T \mathbb{H}^{2} \cong \mathcal{H}^{2} \cong \mathbb{E}_{1}^{3}$. Here, $\phi_{\widehat{\Gamma}}(t, u)$ is the time-like ruled surface in $\mathbb{E}_{1}^{3}$ corresponding to the space-like curve $\widehat{\Gamma}(t)=\gamma(t)+\varepsilon \vec{\nu}(t)$ (or to the smooth curve $\Gamma(t) \in T \mathbb{H}^{2}$ ) on $\mathcal{H}_{0}^{2}$ having the base curve

$$
\widehat{\beta}(t)=\vec{\gamma}(t) \wedge_{1} \vec{\nu}(t)
$$




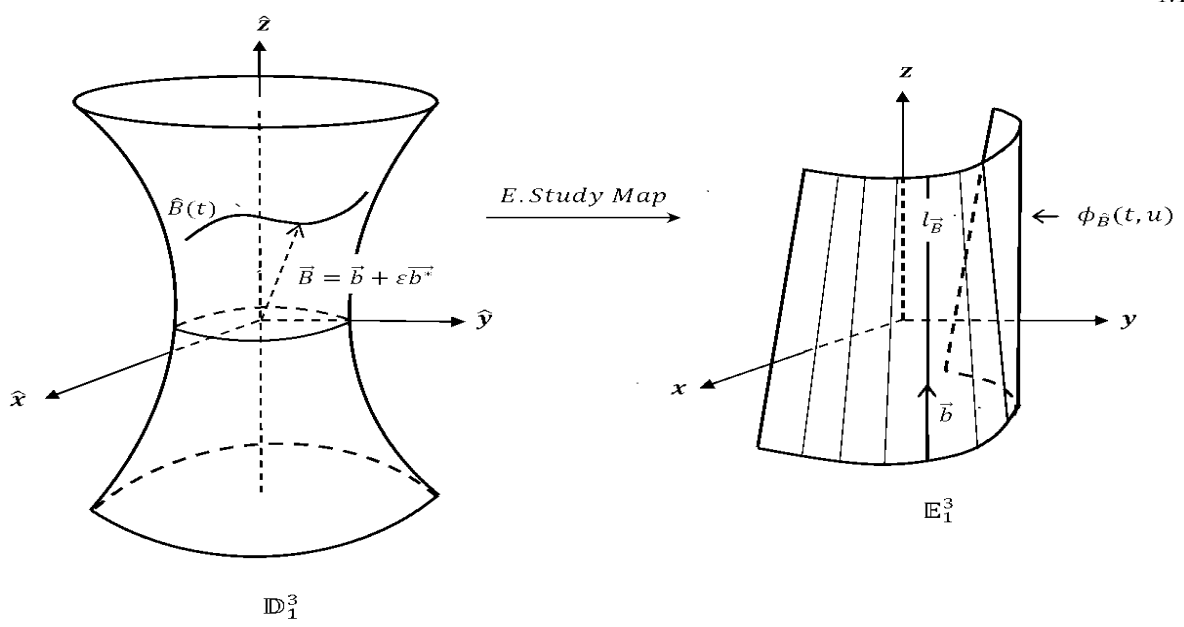

Fig. 4: The curve $\widehat{B}(t)$ on $\mathcal{S}_{1}^{2}$ and the corresponding ruled surface $\phi_{\widehat{B}}(t, u)$ in $\mathbb{E}_{1}^{3}$.

The map

$$
T \mathbb{S}_{1}^{2} \rightarrow \mathcal{S}_{1}^{2} \rightarrow \mathbb{E}_{1}^{3}
$$

defined by

$$
\Gamma(t)=(\gamma(t), \nu(t)) \mapsto \widehat{\Gamma}(t)=\vec{\gamma}(t)+\varepsilon \vec{\nu}(t) \rightarrow \phi_{\widehat{\Gamma}}(t, u)=\vec{\gamma}(t) \wedge_{1} \vec{\nu}(t)+u \vec{\gamma}(t)
$$

is an isomorphism, i.e. $T \mathbb{S}_{1}^{2} \cong \mathcal{S}_{1}^{2} \cong \mathbb{E}_{1}^{3}$. Here, $\phi_{\widehat{\Gamma}}(t, u)$ is the space-like (resp. time-like) ruled surface in $\mathbb{E}_{1}^{3}$ corresponding to the time-like (resp. space-like) curve $\widehat{\Gamma}(t)=\gamma(t)+\varepsilon \vec{\nu}(t)$ (or to the smooth curve $\left.\Gamma(t) \in T \mathbb{S}_{1}^{2}\right)$ on $\mathcal{S}_{1}^{2}$ having the base curve

$$
\widehat{\beta}(t)=\vec{\gamma}(t) \wedge_{1} \vec{\nu}(t)
$$

By differentiating the base curve $\widehat{\beta}(t)=\vec{\gamma}(t) \wedge_{1} \vec{\nu}(t)$, we get

$$
\widehat{\beta}^{\prime}(t)=\vec{\gamma}^{\prime}(t) \wedge_{1} \vec{\nu}(t)+\vec{\gamma}(t) \wedge_{1} \vec{\nu}^{\prime}(t)
$$

and by taking account that $\vec{\gamma}(t)$ is a unit, we get the developability condition for the ruled surface

$$
\phi_{\widehat{\Gamma}}(t, u)=\vec{\gamma}(t) \wedge_{1} \vec{\nu}(t)+u \vec{\gamma}(t)
$$

as

$$
\operatorname{det}\left(\widehat{\beta}^{\prime}(t), \vec{\gamma}(t), \vec{\gamma}^{\prime}(t)\right)=\left\langle\vec{\gamma}^{\prime}(t), \vec{\nu}^{\prime}(t)\right\rangle_{1}=0
$$

Corollary 3.4. Let $\widehat{\Gamma}(t)=\vec{a}(t)+\varepsilon \overrightarrow{a^{*}}(t)$ be a space-like curve on $\mathcal{S}_{1}^{2}$ (resp. time-like on $\mathcal{H}^{2}$ ). Then, the ruled surface $\Phi(t, u)$ corresponding to $\widehat{\Gamma}(t)$ in $\mathbb{E}_{1}^{3}$ is developable if and only if

$$
<\vec{a}^{\prime}(t), \vec{a}^{* \prime}(t)>_{1}=0 .
$$

The proof is a direct consequence of Eq. (9).

Let $\widehat{\Gamma}(t)=\vec{a}(t)+\varepsilon \overrightarrow{a^{*}}(t)$ be a dual curve on $\mathcal{S}_{1}^{2}\left(\right.$ resp. $\left.\mathcal{H}^{2}\right)$. Then, the ruled surface $\Phi(t, u)$ corresponding to $\widehat{\Gamma}(t)$ in $\mathbb{E}_{1}^{3}$ is developable if and only if $\widehat{\Gamma}(t)$ and $\vec{a}(t)$ have the same arc-length parameter. 
Let $\widehat{\Gamma}(t)=\vec{a}(t)+\varepsilon \overrightarrow{a^{*}}(t)$ be a dual curve on $\mathcal{S}_{1}^{2}$ or $\mathcal{H}^{2}$. Then the norm of $\widehat{\Gamma}(t)$ is

$$
\begin{aligned}
\|\widehat{\Gamma}(t)\|_{1} & =\left\|\vec{a}(t)+\varepsilon \vec{a}^{*}(t)\right\|_{1} \\
& =\sqrt{\left|\left\langle\vec{a}^{\prime}(t), \vec{a}^{\prime}(t)\right\rangle_{1}+2 \varepsilon\left\langle\vec{a}^{\prime}(t), \vec{a}^{* \prime}(t)\right\rangle_{1}\right|} \\
& =\sqrt{\left|\left\langle\vec{a}^{\prime}(t), \vec{a}^{\prime}(t)\right\rangle_{1}\right|}+\varepsilon \frac{\left\langle\vec{a}^{\prime}(t), \vec{a}^{* \prime}(t)\right\rangle_{1}}{\sqrt{\left|\left\langle\vec{a}^{\prime}(t), \vec{a}^{\prime}(t)\right\rangle_{1}\right|}}
\end{aligned}
$$

The arc-length parameter $\hat{t}$ is

$$
\hat{t}=\int_{0}^{t}\|\widehat{\Gamma}(t)\|_{1} d s=t+\varepsilon \int_{0}^{t} \frac{\left\langle\vec{a}^{\prime}(t), \vec{a}^{* \prime}(t)\right\rangle_{1}}{\sqrt{\left\langle\vec{a}^{\prime}(t), \vec{a}^{\prime}(t)\right\rangle_{1}}} d s
$$

From the developability condition $\left\langle\vec{a}^{\prime}(t), \vec{a}^{* \prime}(t)\right\rangle_{1}=0$ for the ruled surface $\Phi(t, u)$ corresponding to $\widehat{\Gamma}(t)$ we get

$$
\hat{t}=t \text {. }
$$

So, $\widehat{\Gamma}(t)$ and $\vec{a}(t)$ have the same arc-length parameter in $\mathcal{S}_{1}^{2}\left(\right.$ resp. $\left.\mathcal{H}^{2}\right)$.

\section{Tangent bundles of $\mathbb{S}_{1}^{2}$ and $\mathbb{H}^{2}$, and ruled surfaces in $\mathbb{E}_{1}^{3}$}

In this section, we give some relationships between "curves in $T \mathbb{S}_{1}^{2}$ and $T \mathbb{H}^{2}$ " and "their corresponding ruled surfaces in $\mathbb{E}_{1}^{3}$ ". Moreover, the developability conditions of these ruled surfaces are investigated.

Let $\Gamma(t)=(\gamma(t), v(t))$ be a smooth curve in $T \mathbb{S}_{1}^{2}$ (resp. $\left.T \mathbb{H}^{2}\right)$. Then, the space-like or time-like (resp. space-like) ruled surface $\Phi(t, u)$ corresponding to $\Gamma$ in $\mathbb{E}_{1}^{3}$ is developable if and only if

$$
\left\langle\gamma^{\prime}(t), v^{\prime}(t)\right\rangle_{1}=0
$$

The proof can be given straightforward by using Eq. (9) and Cor. (3.4).

Example 4.1. Let $\gamma: I \subset \mathbb{R} \rightarrow \mathbb{S}_{1}^{2}$ (resp. $\mathbb{H}^{2}$ ) be a smooth curve with the Frenet frame apparatus $\{T, N, B, \kappa, \tau\}$ and $\lambda$ be a real constant. Then, the curve

$$
\Gamma=(T, \lambda N)
$$

is in $T \mathbb{S}_{1}^{2}$ (resp. $\mathbb{H}^{2}$ ) and from Eq. (3), we have

$$
\begin{aligned}
T^{\prime}(t) & =\kappa(t) N(t) \text { and }(\lambda N)^{\prime}(t)=-\lambda \varepsilon_{1} \kappa(t) T(t)+\lambda \tau(t) B(t) \\
\text { resp. } T^{\prime}(t) & \left.=\kappa(t) N(t) \text { and }(\lambda N)^{\prime}(t)=\lambda \kappa(t) T(t)+\lambda \tau(t) B(t)\right)
\end{aligned}
$$

and thus

$$
\left\langle T^{\prime}(t),(\lambda N)^{\prime}(t)\right\rangle_{1}=0 .
$$

Hence, the ruled surface $\Phi(t, u)$ corresponding to $\Gamma$ in $\mathbb{E}_{1}^{3}$ is developable.

Corollary 4.2. Let $\Gamma=(\gamma, v)$ be a smooth curve in $T \mathbb{S}_{1}^{2}$ (resp. THتH$\left.{ }^{2}\right)$. Then, the couple $(\gamma, v)$ is an involuteevolute curve couple if and only the space-like or time-like (resp. space-like) ruled surface $\Phi$ corresponding to $\Gamma$ in $\mathbb{E}_{1}^{3}$ is developable.

Corollary 4.3. Let $\Gamma=(\gamma, v)$ be a smooth curve in $T \mathbb{S}_{1}^{2}$ (resp. $\left.T \mathbb{H}^{2}\right)$. If the couple $(\widehat{\beta}, \gamma)$ is an involute-evolute curve couple, then the striction curve $\bar{\beta}(t)$ and the base curve $\widehat{\beta}(t)$ of the space-like or time-like (resp. space-like) ruled surface $\Phi$ corresponding to $\Gamma$ in $\mathbb{E}_{1}^{3}$ coincide. 
Example 4.4. Let $\gamma: I \subset \mathbb{R} \rightarrow \mathbb{H}^{2}$ be a smooth curve with frame apparatus $\{N, C, W, f, g\}$ and $\lambda$ be a real constant, see [2]. The curve

$$
\Gamma=(N, \lambda W)
$$

is in $T \mathbb{H}^{2}$ and from [2] we have

$$
N^{\prime}(t)=f(t) C(t) \text { and }(\lambda W)^{\prime}(t)=-\lambda f(t) C(t) .
$$

Hence, we obtain

$$
\left\langle N^{\prime}(t),(\lambda C)^{\prime}(t)\right\rangle_{1}=-\lambda(f g)(t) .
$$

From Cor. (3.4), the couple $(N, \lambda W)$ is an involute-evolute curve couple if and only if $(f g)(t)=0$. In this case, the ruled surface $\Phi(t, u)$ corresponding to $\Gamma$ in $\mathbb{E}_{1}^{3}$ is developable which means that

$$
\frac{\tau}{\kappa} \text { is constant }
$$

where

$$
f=\sqrt{\left|\kappa^{2}-\tau^{2}\right|} \quad \text { and } \quad g=f \frac{\kappa^{2}}{\left(\left|\kappa^{2}-\tau^{2}\right|\right)^{\frac{3}{2}}}\left(\frac{\tau}{\kappa}\right)^{\prime} .
$$

Here, $\kappa$ and $\tau$ are the curvatures functions of $\gamma$. So, the curve $\gamma$ is a general helix in $\mathbb{E}_{1}^{3}$. Similary, we find a same result if the curve $\gamma$ is in $\mathbb{S}_{1}^{2}$.

\subsection{Unit tangent bundle of $\mathbb{S}_{1}^{2}$ and $\mathbb{H}^{2}$, and ruled surfaces in $\mathbb{E}_{1}^{3}$}

In this section, we give some relationships between "curves in $U T \mathbb{S}_{1}^{2}$ and $U T \mathbb{H}^{2}$ " and "their corresponding ruled

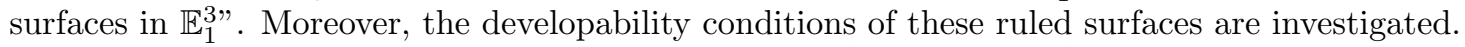

Let $\Gamma(t)=(\gamma(t), v(t))$ be a smooth curve in $U T \mathbb{S}_{1}^{2}$ (resp. $U T \mathbb{H}^{2}$ ), then we have $\langle\gamma(t), v(t)\rangle_{1}=0$. By taken $\mu(t)=\gamma(t) \wedge v(t)$, the moving frame $\{\gamma(t), v(t), \mu(t)\}$ is called the Frenet frame along $\gamma(t)$. The Frenet formulae of this moving frame can be given by

$$
\begin{gathered}
\left(\begin{array}{c}
\gamma^{\prime}(t) \\
v^{\prime}(t) \\
\mu^{\prime}(t)
\end{array}\right)=\left(\begin{array}{ccc}
0 & l(t) & m(t) \\
-\varepsilon_{1} l(t) & 0 & n(t) \\
-m(t) & \varepsilon_{1} n(t) & 0
\end{array}\right)\left(\begin{array}{c}
\gamma(t) \\
v(t) \\
\mu(t)
\end{array}\right) \\
\text { (resp. } \left.\left(\begin{array}{c}
\gamma^{\prime}(t) \\
v^{\prime}(t) \\
\mu^{\prime}(t)
\end{array}\right)=\left(\begin{array}{ccc}
0 & l(t) & m(t) \\
l(t) & 0 & n(t) \\
-m(t) & -n(t) & 0
\end{array}\right)\left(\begin{array}{l}
\gamma(t) \\
v(t) \\
\mu(t)
\end{array}\right)\right)
\end{gathered}
$$

where $\left\langle\gamma^{\prime}(t), \gamma^{\prime}(t)>_{1}=1\right.$ (resp. $\left\langle\gamma^{\prime}(t), \gamma^{\prime}(t)>_{1}=-1\right),<v(t), v(t)>_{1}=\varepsilon_{1}= \pm 1\left(\operatorname{resp} .<v(t), v(t)>_{1}=1\right)$, $\langle\mu(t), \mu(t)\rangle_{1}=-\varepsilon_{1}$ (resp. $\|\mu(t)\|_{1}=1$ ), $l(t)=\left\langle\gamma^{\prime}(t), v(t)\right\rangle_{1}, m(t)=\left\langle\gamma^{\prime}(t), \mu(t)\right\rangle_{1}$ and $n(t)=\left\langle v^{\prime}(t), \mu(t)\right\rangle_{1}$. We call the triple $(l, m, n)$ the curvature functions of the curve $\Gamma$ which depends on the parametrization.

Remark 4.5. Let $\Gamma(t)=(\gamma(t), v(t))$ be a curve in $T \mathbb{S}_{1}^{2}$ or $T \mathbb{H}^{2}$ (that means, $\left.\langle\gamma(t), v(t)\rangle_{1}=0\right)$. If we take $l(t)=0$, then $\Gamma(t)$ is a Legendre curve.

Let $\Gamma(t)=(\gamma(t), v(t))$ be a smooth curve in $U T \mathbb{S}_{1}^{2}$ or $U T \mathbb{H}^{2}$ and $\Phi(t, u)=\gamma(t) \wedge_{1} \nu(t)+u \gamma(t)$ be the corresponding ruled surface to $\Gamma(t)$ in $\mathbb{E}_{1}^{3}$, then we obtain

$$
\begin{aligned}
\Phi_{t}(t, u) & =\gamma^{\prime}(t) \wedge_{1} v(t)+\gamma(t) \wedge_{1} v^{\prime}(t)+u \gamma^{\prime}(t) \\
& =-m(t) \gamma(t)+n(t) v(t)+u \gamma^{\prime}(t) \\
\Phi_{u}(t, u) & =\gamma(t) \\
\Phi_{t}(t, u) \wedge_{1} \Phi_{u}(t, u) & =u m(t) v(t)+(u l(t)-n(t)) \mu(t) .
\end{aligned}
$$

The normal vector of the ruled surface $\Phi(t, u)$ is

$$
N_{\Phi}=\frac{\Phi_{t}(t, u) \wedge_{1} \Phi_{u}(t, u)}{\left\|\Phi_{t}(t, u) \wedge_{1} \Phi_{u}(t, u)\right\|_{1}}=\frac{(u m(t) v(t)+(u l(t)-n(t)) \mu(t))}{\|u m(t) v(t)+(u l(t)-n(t)) \mu(t)\|_{1}} .
$$


Let $\Gamma=(\gamma, v)$ be a smooth curve in $T \mathbb{S}_{1}^{2}$ or $T \mathbb{H}^{2}$. Then, the causal character of the corresponding ruled surface $\Phi$ to $\Gamma$ in $\mathbb{E}_{1}^{3}$ depends on the causal characters of the vectors $v$ and $\mu$.

Applying Prop. (4) and Frenet frame given by Eq. (11), we obtain the developability condition and the causal characters of the ruled surface $\Phi(t, u)$ with the moving frame $\{\gamma(t), v(t), \mu(t)\}$ as

$$
\left\langle\gamma^{\prime}(t), v^{\prime}(t)\right\rangle_{1}=m(t) n(t)=0 \quad \text { for all } t \in I
$$

and we can give the following propositions;

Let $\Gamma=(\gamma, v)$ be a Legendre curve in $U T \mathbb{S}_{1}^{2}$ (resp. $\left.U T \mathbb{H}^{2}\right)$. If the couple $(\gamma, v)$ is an involute-evolute couple, then the ruled surface $\Phi$ corresponding to $\Gamma$ in $\mathbb{E}_{1}^{3}$ is a developable surface.

Let $\Gamma=(\gamma, v)$ be a smooth curve in $U T \mathbb{S}_{1}^{2}$ (resp. $\left.U T \mathbb{H}^{2}\right)$. Then, the ruled surface $\Phi$ corresponding to $\Gamma$ in $\mathbb{E}_{1}^{3}$ is developable if and only if $\Phi$ is a cylinder (resp. space-like cylinder) with different causal character to $v$ or a plane with the same causal character to $v$ (resp. space-like plane).

For the Legendre curve $\Gamma(t)=(\gamma(t), v(t))$ in $U T \mathbb{S}_{1}^{2}$, the developability condition is

$$
\left\langle\gamma^{\prime}(t), v^{\prime}(t)\right\rangle_{1}=m(t) n(t)=0 \quad \text { for all } t \in I
$$

Thus, we have the following two cases $m(t)=0$ or $n(t)=0$.

(i) If $m(t)=0$, using the Eq. (11) and Eq. (12), we obtain $\gamma^{\prime}(t)=0$ and $\left\|N_{\Phi}\right\|_{1}=\|\mu\|_{1}$. That means, $\gamma$ is a constant and $\Phi$ is a cylinder with different causal character to $v$.

(ii) If $n(t)=0$, using the Eq. (11) and Eq. $\left[12\right.$, we obtain $v^{\prime}(t)=0$ and $\left\|N_{\Phi}\right\|_{1}=v$. That means, $v(t)$ is a constant. So, the ruled surface $\Phi(t, u)$ is a plane with the same causal character to $v$.

Similarly. we can prove the case $U T \mathbb{H}^{2}$.

Theorem 4.6. Let $\Gamma=(\gamma, v)$ be a smooth curve in $U T \mathbb{S}_{1}^{2}$ (resp. UTH $\mathbb{H}^{2}$ ) and $\Phi$ be the corresponding surface to $\Gamma$ in $\mathbb{E}_{1}^{3}$. The striction curve $\bar{\beta}$ and the base curve $\widehat{\beta}$ of $\Phi$ coincide if and only if the non-constant curve $\gamma$ is Legendre or the ruled surface $\Phi$ is a plane with the same causal character to $v$ (resp. space-like plane).

Let $\Gamma(t)=(\gamma(t), v(t)) \in U T \mathbb{S}_{1}^{2}$ (resp. $U T \mathbb{H}^{2}$ ) be a smooth curve. Using Eq. (6) and the moving frame $\{\gamma(t), v(t), \mu(t)\}$ given by Eq. 11 , we have

$$
\bar{\beta}(t)=\beta(t) \text { for all } t \in I
$$

if and only if

$$
\left\langle T_{\beta}, \gamma^{\prime}(t)\right\rangle_{1}=\left\langle\beta^{\prime}(t), \gamma^{\prime}(t)\right\rangle_{1}=l(t) n(t)=0
$$

Hence the following two cases can be given:

(i) If $l(t)=0$, from Rem. 4.5 we can say that $\Gamma(t)$ is Legendre in $T \mathbb{S}_{1}^{2}$ (resp. $T \mathbb{H}^{2}$ ), i.e. $\Gamma(t)$ is Legendre in $U T \mathbb{S}_{1}^{2}\left(\right.$ resp. $\left.U T \mathbb{H}^{2}\right)$.

(ii) If $n(t)=0$, then from Prop.4.1), $\Phi(t, u)$ is a plane with the same causal character to $v$ (resp. space-like plane).

A smooth curve $\Gamma=(\gamma, v)$ is a curve in $T \mathbb{S}_{1}^{2}$ (resp. $\left.U T \mathbb{H}_{1}^{2}\right)$ if and only if the base curve $\widehat{\beta}(t)$ of the ruled surface $\Phi(t, u)$ corresponding to $\Gamma$ in $\mathbb{E}_{1}^{3}$ is in $\mathbb{S}_{1}^{2}$ or $\mathbb{H}^{2}$ (resp. $\mathbb{H}^{2}$ ).

Let $\Gamma=(\gamma, v)$ be a smooth curve in $T \mathbb{S}_{1}^{2}\left(\right.$ resp. $\left.U T \mathbb{H}_{1}^{2}\right)$, then

$$
\begin{aligned}
<\widehat{\beta}(t), \widehat{\beta}(t)>_{1}=<\gamma(t), \gamma(t)>_{1}<v(t), v(t)>_{1}= \pm 1 \\
\left(\text { resp. } \quad<\widehat{\beta}(t), \widehat{\beta}(t)>_{1}=<\gamma(t), \gamma(t)>_{1}<v(t), v(t)>_{1}=-1\right)
\end{aligned}
$$

So, $\widehat{\beta}(t)$ is a curve in $\mathbb{S}_{1}^{2}$ or $\mathbb{H}^{2}$ (resp. $\left.\mathbb{H}^{2}\right)$.

Corollary 4.7. Let $\Gamma=(\gamma, v)$ be a smooth curve in $T \mathbb{S}_{1}^{2}$ (resp. UTHتH $\mathbb{H}_{1}^{2}$ ). If $\Gamma$ is a Legendre curve, then the striction curve $\bar{\beta}$ of the ruled surface $\Phi$ is in $\mathbb{S}_{1}^{2}$ or $\mathbb{H}^{2}$ (resp. $\mathbb{H}^{2}$ ). 
If $\Gamma=(\gamma, v)$ is a Legendre curve in $U T \mathbb{S}_{1}^{2}$ or $U T \mathbb{H}^{2}$, then $l(t)=0$ and from Eq. [11), we have

$$
\begin{aligned}
\gamma^{\prime}(t) & =m(t) \mu(t) \\
\gamma^{\prime \prime}(t) & =m^{\prime}(t) \mu(t)+m(t) \mu^{\prime}(t) \\
\gamma^{\prime}(t) \wedge_{1} \gamma^{\prime \prime}(t) & =-\varepsilon_{1} m^{2}(t) n(t) \gamma(t)-m^{3}(t) v(t) .
\end{aligned}
$$

The normal curvature of $\gamma$ is

while its geodesic curvature is

$$
\kappa_{\gamma}=\frac{\left\|\gamma^{\prime}(t) \wedge_{1} \gamma^{\prime \prime}(t)\right\|_{1}}{\left\|\gamma^{\prime}(t)\right\|_{1}^{3}}=\sqrt{1-\left(\frac{n(t)}{m(t)}\right)^{2}}
$$

$$
\kappa_{g}=\sqrt{1-\kappa_{\gamma}^{2}}=\frac{n(t)}{m(t)} .
$$

Theorem 4.8. Let $\gamma$ be a Legendre curve in $U T \mathbb{S}_{1}^{2}$ (resp. UTH $\mathbb{H}^{2}$ ). If $\gamma$ is a geodesic curve, then the ruled surface $\Phi$ corresponding to the smooth curve $\Gamma$ in $\mathbb{E}_{1}^{3}$ is a plane with the same causal character to $v$ (resp. space-like plane).

Let $\gamma$ be a Legendre curve in $U T \mathbb{S}_{1}^{2}$ (resp. $U T \mathbb{H}^{2}$ ). If $\gamma$ is a geodesic curve, i.e. $\kappa_{g}=0$, then using Rem. 4.5 and Eq. 111, we can easily obtain the proof.

Example 4.9. Let $\gamma$ be a curve defined by $\gamma(t)=(0, \sinh t, \cosh t)$, then we have $\langle\gamma(t), \gamma(t)\rangle_{1}=-1$, and thus $\gamma(t) \in \mathbb{H}^{2}$. By taken a vector $v$ defined by $v(t)=(0, t \cosh t, t \sinh t)$ we get $\langle\gamma(t), v(t)\rangle_{1}=0$ that means $\Gamma(t)=$ $(\gamma(t), v(t)) \in T \mathbb{H}^{2}$. The ruled surface $\Phi(t, u)$ corresponding to $\Gamma$ in $\mathbb{E}_{1}^{3}$ may be given as

$$
\Phi(t, u)=\gamma(t) \wedge_{1} v(t)+u \gamma(t)=(-t, u \sinh t, u \cosh t)
$$

having a base curve

$$
\beta(t)=\gamma(t) \wedge_{1} v(t)=(-t, 0,0)
$$

$\Phi(t, u)$ is not developable because $\left\langle\gamma^{\prime}(t) \wedge_{1} v^{\prime}(t)\right\rangle_{1}=1 \neq 0$. Thus, the couple $(\gamma, v)$ is not an involute-evolute couple, see Fig. 5.
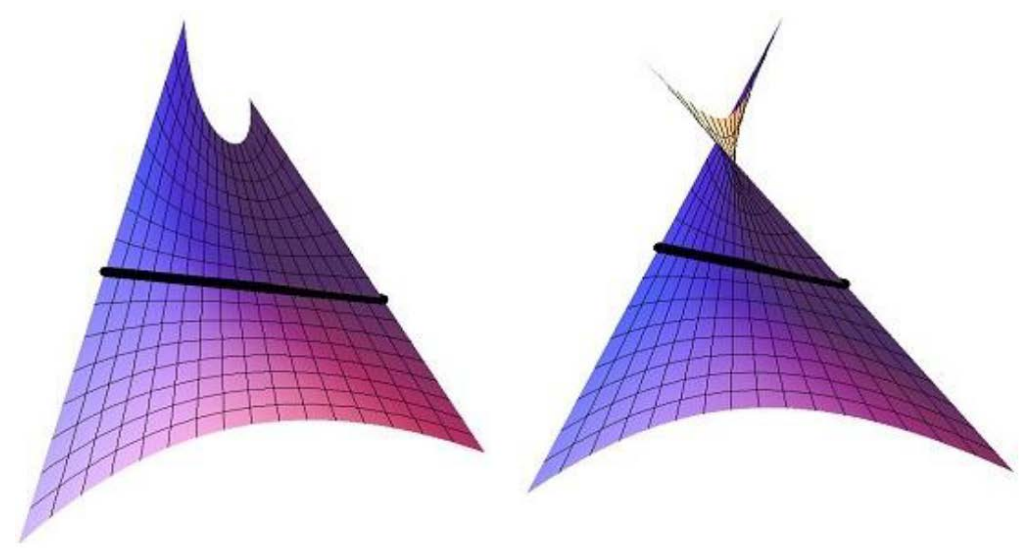

Fig. 5: The ruled surface $\Phi$ with the base curve $\beta$ in $\mathbb{E}_{1}^{3}$.

Example 4.10. Let $\gamma$ be a curve defined by $\gamma(t)=(\cos t, \sin t, 0)$, then we have $\langle\gamma(t), \gamma(t)\rangle_{1}=1$, and thus $\gamma \in \mathbb{S}_{1}^{2}$. By taken a vector $v$ defined by $v(t)=(-t \sin t, t \cos t, 0)$ we get $\langle\gamma(t), v(t)\rangle_{1}=0$ that means $\Gamma(t)=(\gamma(t), v(t)) \in T \mathbb{S}_{1}^{2}$. The ruled surface $\Phi(t, u)$ corresponding to $\Gamma$ in $\mathbb{E}_{1}^{3}$ may be given as

$$
\Phi(t, u)=\gamma(t) \wedge_{1} v(t)+u \gamma(t)=(u \cos t, u \sin t,-t)
$$

having a base curve

$$
\beta(t)=\gamma(t) \wedge_{1} v(t)=(0,0,-t) .
$$

$\Phi(t, u)$ is also not developable because $\left\langle\gamma^{\prime}(t) \wedge_{1} v^{\prime}(t)\right\rangle_{1}=1 \neq 0$. Thus, the couple $(\gamma, v)$ is not an involute-evolute couple, see Fig. 6. 


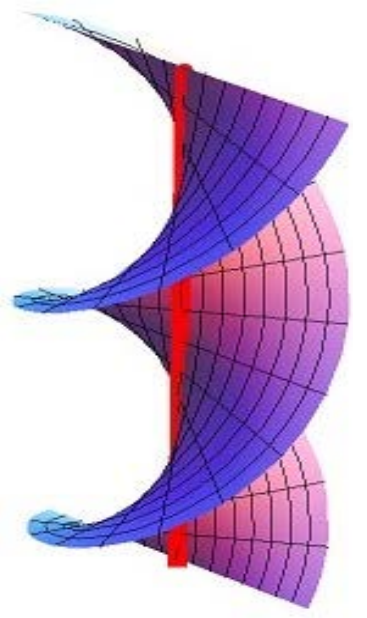

Fig. 6: The ruled surface $\Phi$ with the base curve $\beta$ in $\mathbb{E}_{1}^{3}$.

\section{Conclusion}

In Minkowski 3-space, we have extend the E. Study map to tangent bundles $T \mathbb{S}_{1}^{2}$ and $T \mathbb{H}^{2}$ to show the one-toone correspondence between a smooth curve on $T \mathbb{S}_{1}^{2}$ and $T \mathbb{H}^{2}$, and a space-like or time-like ruled surface in $\mathbb{E}_{1}^{3}$, respectively. We studied the relationships between developability conditions of these ruled surfaces (with different causal characters) and their corresponding smooth curves on $T \mathbb{S}_{1}^{2}$ and $T \mathbb{H}^{2}$ to be Legendre or involute-evolute curve couples.

\section{References}

[1] W. Blaschke. Differential geometrie and geometrischke grundlagen von Einsteins relativitast theorie dover. New York, (1945).

[2] M. Bekar and Y. Yayli. Slant helix curves and acceleration centers in Minkowski 3-space $E_{1}^{3}$. J. Adv. Phys. 6(2017), 133-141. https://doi.org/10.1166/jap.2017.1306

[3] I.S. Fischer. Dual-number methods in kinematics, statics and dynamics. Boca Raton London New York Washington D.C. : CRC Press, (1999). https://doi.org/10.1201/9781315141473

[4] F. Hathout, M. Bekar and Y. Yayli. Ruled surfaces and tangent bundle of unit 2-sphere. Int. J. Geom. Methods Mod. Phys., 14(10)(2017), 1750145 https://doi.org/10.1142/s0219887817501456

[5] R. Lopez. Differential geometry of curves and surfaces in Lorentz-Minkowski space. arXiv: 0810.3351 (2008).

[6] E. Nesovic, U. Ozturk and K. Ilarslan. On ruled surfaces with pseudo null base curve in Minkowski 3-Space. Int. Electron. J. Geom., 9(2)(2016), 9-20.

[7] B. O’Neil. Semi-Riemannian geometry with applications to relativity. Academic Press, New York, (1983). https://doi.org/10.1137/1028086

[8] E. Özbay and M. Oral. A study on rectifying curves in the dual Lorentzian Space. Bull. Korean Math. Soc., 46(5)(2009), 967-978. https://doi.org/10.4134/bkms.2009.46.5.967

[9] E. Study. Geometrie der dynamen. Leibzig, (1903).

[10] D.J. Struik. Lectures on classical differential geometry. Addison-Wesley Publishing Company, Inc., (1961).

[11] H.H. Uğurlu and A. Çalışkan. The study mapping for directed spacelike and timelike lines in Minkowski 3-space $R_{1}^{3}$. Math. Comput. Appl., 1(2)(1996), 142-148. 
[12] Y. Yayli, A. Caliskan and H.H. Ugurlu. The E. Study mapping of circles on dual hyperbolic and Lorentzian unit spheres $H_{0}^{2}$ and $S_{1}^{2}$. Math. Proc. Roy. Irish Acad., 102(A1)(2012), 37-47.

https://doi.org/10.3318/pria.2002.102.1.37 